ESAIM: M2AN 51 (2017) 427-442

DOI: $10.1051 / \mathrm{m} 2 \mathrm{an} / 2016041$
ESAIM: Mathematical Modelling and Numerical Analysis

www.esaim-m2an.org

\title{
ON NONTRADITIONAL QUASI-GEOSTROPHIC EQUATIONS
}

\author{
Carine Lucas ${ }^{1}$, James C. McWilliams ${ }^{2}$ and Antoine Rousseau ${ }^{3}$
}

\begin{abstract}
In this article, we work on nontraditional models where the so-called traditional approximation on the Coriolis force is removed. In the derivation of the quasi-geostrophic equations, we carefully consider terms in $\delta / \varepsilon$, where $\delta$ (aspect ratio) and $\varepsilon$ (Rossby number) are both small numbers. We provide here some rigorous crossed-asymptotics with regards to these parameters, prove some mathematical results and compare QHQG and QG models.
\end{abstract}

Mathematics Subject Classification. 35Q35, 76U05, 76M45, 35B40.

Received November 12, 2015. Revised May 5, 2015. Accepted May 30, 2016.

\section{INTRODUCTION}

Equations used to model oceans rely on several hypotheses, depending on the underlying accuracy requirements in numerical simulations. One of these hypotheses is the so-called traditional approximation, which consists in neglecting the cosine part of the Coriolis force, modifying the zonal and vertical components of the momentum equations (see $f^{*}$ terms in Eqs. (2.1a) and (2.1c)). This approximation has been widely discussed in the literature (see [9] and the correspondence in [20,21,26,28]).

Discussions on the traditional approximation usually appear when studying oceans near the equator. Indeed, the Coriolis force expresses as $\vec{\Omega} \times U$ where $U$ is the fluid velocity, and $\vec{\Omega}=\Omega^{t}(0, \cos \theta, \sin \theta), \theta$ being the latitude. When modeling ocean in the equatorial zone, the latitude becomes smaller and the cosine part of the Coriolis force is not negligible compared to the sine part. This is the approach developed for example in [6], based on [27], or in [22], where the author refers to [29] to assert that the $\cos \theta$ Coriolis terms cannot be omitted.

These cosine terms are traditionally not considered in the quasi-geostrophic equations that are very familiar to oceanographers and meteorologists. These equations have been extensively used for modeling oceanic and atmospheric circulations $[3,4]$. A way to obtain the quasi-geostrophic model is to perform an asymptotic expansion, with respect to the Rossby number, in the 3D primitive (hydrostatic) equations (PEs). The model is also very familiar to applied mathematicians, and several studies establishing the well-posedness of the corresponding boundary-value problem have been published (see e.g., $[2,13,18]$ ). Here, as we do not perform the traditional

\footnotetext{
Keywords and phrases. Ocean modeling, Coriolis force, traditional approximation, tilted quasi-geostrophic equations, slanted rotation.

1 MAPMO UMR CNRS 7349, Fédération Denis Poisson FR CNRS 2964, Université d'Orléans, 45067 Orléans cedex 2 , France. Carine.Lucas@univ-orleans.fr

2 Dept. of Atmospheric and Oceanic Sciences, University of California, Los Angeles (UCLA), Mathematical Sciences Building, Room 7983, Los Angeles, CA 90095-1565. USA. jcm@atmos.ucla.edu

3 Inria and IMAG UMR CNRS 5159, Inria Chile, Av Apoquindo 2827, Las Condes, Santiago de Chile, Chile.

Antoine.Rousseau@inria.fr
} 
approximation of the Coriolis force, we shall start this paper with the quasi-hydrostatic PEs (2.1) (see [15] for a mathematical study).

As mentioned by some of the authors in [16] for the shallow water equations and in [15] for the 3D primitive equations, taking the cosine terms into account leads to a second order correction of the traditional hydrostatic approximation, towards non-hydrostatic modeling: hence the naming "quasi-hydrostatic" (QH) ocean modeling. Even if it was not named that way in the literature, the quasi-hydrostatic quasi-geostrophic model (QHQG) has already been studied by several authors: see for example the so-called tilted QG in [14], with formal derivation but no well-posedness result, or the slanted model in [11] in which small Rossby asymptotics are analyzed by a fast wave averaging approach.

A key parameter in the following is the nontraditional parameter $\lambda=\delta \cot \theta$ where $\delta=H / L$ is the domain aspect ratio. In most cases (ocean modeling away from the equator), $\lambda$ is small. But when approaching the equator and/or in cases where the typical aspect ratio is not small, nontraditional models should rather be considered.

In this work we have a twofold objective:

- show that the derivation of the (nontraditional) QHQG model and its well-posedness do not raise any additional difficulty, compared to traditional QG. For this reason, we will mimic the reasoning of the reference papers $[1,2]$ and shall underline the (few) necessary adjustments required for the QH regime. The authors believe that this should be a key argument to prefer QHQG rather than QG: indeed, physics is more accurate while theoretical results can be extended from QG to QHQG (as for HPE vs QHPE, see [15]). This is the objective of Section 2;

- position the so-called QHQG equations in the framework of geostrophic and hydrostatic regimes. Looking at the scheme of Figure 2, one can see that the geostrophic regime $(\varepsilon \rightarrow 0)$ has been studied in the literature both in the traditional (see (2), bottom part of Fig. 2, e.g. [2] and [5]) and nontraditional (see (1), top of Fig. 2, e.g. [11]) configurations. In Section 3 below, we show that solutions of the QHQG model converge to solutions of the QG model as $\lambda=\delta \cot \theta$ goes to zero. In other words, we claim that QG is a relevant approximation of QHQG when $\lambda$ is a small parameter.

\section{QuAsi-Geostrophic EQUations}

Since the QG equations are obtained in the zero-limit of the Rossby number (large rotational effects), we choose to retain all rotating terms in the original equations, before performing the asymptotic analysis. We will see in equation (2.16) that the modified QG equations, that we will call quasi-hydrostatic quasi-geostrophic (QHQG) equations, are very similar to the traditional QG ones, except that they raise a new vertical direction (denoted $Z$ hereafter), which differs from the traditional vertical direction $z$, see also [14]. The tilt between $z$ and $Z$ is proportional to the nondimensional parameter $\lambda$ introduced in Section 2.1.1, which mesures the ratio between traditional and nontraditional Coriolis terms. Experimental and numerical evidences of this tilted vertical direction can be found in $[12,24,25,31]$.

\subsection{Derivation of the QHQG model}

In this section we present the derivation of the quasi-hydrostatic quasi-geostrophic (QHQG) equations. The derivation follows classical principles (as in [2]): scaling, asymptotic expansion with respect to a small parameter, equations satisfied at order zero and one. Here, the small parameter (denoted $\varepsilon$ in the sequel) is the Rossby number, so that we underline the effect of rotating terms. In order to account for the complete Coriolis force (see e.g., [15] and references therein), we retain all the rotating terms in the original equations, including the terms that are usually neglected in the so-called traditional approximation. This denomination was introduced by Carl Eckart [9]. 


\subsubsection{Scaling parameters and scaled equations}

We consider a three-dimensional domain with periodic boundary conditions in the horizontal directions, rigid lid and flat bottom in the vertical. The governing non-hydrostatic equations, including the complete Coriolis force, read:

$$
\begin{aligned}
\frac{D u}{D t}-f v+f^{*} w & =-\partial_{x} \varphi+F_{u}, \\
\frac{D v}{D t}+f u & =-\partial_{y} \varphi+F_{v}, \\
\frac{D w}{D t}-f^{*} u+\frac{g \varrho}{\varrho_{0}} & =-\partial_{z} \varphi+F_{w}, \\
\partial_{x} u+\partial_{y} v+\partial_{z} w & =0, \\
\frac{D \varrho}{D t} & =F_{\varrho} .
\end{aligned}
$$

Here $(u, v, w)$ and $\varrho$ are respectively the three-dimensional velocity and density of the fluid, $\varrho_{0}$ stands for the averaged density of the fluid, and $\varphi$ is the renormalized pressure, $\varphi=p / \varrho_{0}$. The scalars $f=2 \Omega \sin (\theta)$ and $f^{*}=2 \Omega \cos (\theta)$ are the Coriolis parameters where $\Omega$ stands for the angular velocity of the earth and $\theta$ is the latitude; $g$ is the universal gravity constant. The terms $F_{\bullet}$ correspond to external forces (including diffusion terms).

Equations (2.1a) to (2.1c) describe the conservation of momentum, where $D / D t$ is the material derivative $D / D t=\partial_{t}+u \partial_{x}+v \partial_{y}+w \partial_{z}$, and equation (2.1d) corresponds to the conservation of mass. Finally, equation $(2.1 \mathrm{e})$ describes the advection of tracers (here the density $\varrho$ ). The density and the pressure may be classically decomposed as

$$
\varrho(x, y, z, t)=\bar{\rho}(z)+\rho(x, y, z, t)
$$

and

$$
\varphi(x, y, z, t)=\bar{\varphi}(z)+\phi(x, y, z, t),
$$

where $\bar{\rho}$ and $\bar{\varphi}$ are the (known) background density and potential, depending only on the vertical variable. The functions $\bar{\rho}$ and $\bar{\varphi}$ are in hydrostatic balance $\left(\partial_{z} \bar{\varphi}=-\bar{\rho} g\right)$. We also denote by $N^{2}(z)=-\partial_{z} \bar{\rho}(z)$ the buoyancy frequency, assuming that $\partial_{z} \bar{\rho}$ is negative and bounded away from zero.

Before going further in the derivation of the corresponding QG model, let us insist on the fact that we keep in equations (2.1a) and (2.1c) the Coriolis terms $f^{*} w$ and $f^{*} u$ : we do not use the traditional approximation. We will finally lead to a slightly modified QG model (see Eq. (2.16)). We think that it is a relevant modification, since the QG approximation aims at underlying the Earth's rotation effects: one should thus include every rotation term in the primitive equations prior to an asymptotic expansion with respect to the Rossby number.

In the context of the $\beta$-plane approximation (see [8] for example), as suggested in [12] we have, with $\theta_{0}$ the average latitude and $R_{e}$ the mean radius of the Earth:

$$
\begin{gathered}
f=f_{0}+\frac{1}{R_{e}} 2 \Omega \cos \theta_{0} y, \text { with } f_{0}=2 \Omega \sin \theta_{0}, \\
f^{*}=f_{0}^{*}=2 \Omega \cos \theta_{0} .
\end{gathered}
$$

We now introduce the following dimensionless variables, as it is classically done in QG modeling:

$$
\begin{array}{r}
(x, y)=L\left(x^{\prime}, y^{\prime}\right), \quad z=H z^{\prime}, \quad t=\frac{L}{U} t^{\prime}, \\
u=U u^{\prime}, \quad v=U v^{\prime}, \quad w=\frac{U H}{L} w^{\prime}, \\
\bar{\rho}=P \bar{\rho}^{\prime} \text { and } \bar{\varphi}=H P g \bar{\varphi}^{\prime}, \quad \rho=\frac{\varrho_{0} f_{0} U L}{g H} \rho^{\prime}, \quad \phi=f_{0} U L \phi^{\prime} .
\end{array}
$$


The Rossby number $\varepsilon=U / f_{0} L$ is the fundamental ordering parameter in the following asymptotic expansion. A secondary ordering parameter is the scale ratio of $\rho$ to $\varrho$ : this ratio is assumed to be $\varepsilon$ as usual for large scale oceanography, that is ${ }^{4}$, we assume:

$$
\frac{\varrho_{0} f_{0} U L}{g H}=P \varepsilon .
$$

Finally, the density may be expressed in terms of nondimensionnal quantities:

$$
\varrho=P\left(\bar{\rho}^{\prime}(z)+\varepsilon \rho^{\prime}\right),
$$

as well as the Coriolis parameter:

$$
f=f_{0}\left(1+\varepsilon \beta_{0} y^{\prime}\right) \quad \text { with } \beta_{0}=\frac{2 \Omega L^{2} \cos \theta_{0}}{U R_{e}} .
$$

We end this section with the non-hydrostatic scaled equations (we naturally drop the primes):

$$
\begin{aligned}
\frac{D u}{D t}-\left(\frac{1}{\varepsilon}+\beta_{0} y\right) v+\frac{\lambda}{\varepsilon} w & =-\frac{1}{\varepsilon} \partial_{x} \phi+\nu_{1 h} \Delta_{h} u+\nu_{1 z} \partial_{z z}^{2} u \\
\frac{D v}{D t}+\left(\frac{1}{\varepsilon}+\beta_{0} y\right) u & =-\frac{1}{\varepsilon} \partial_{y} \phi+\nu_{1 h} \Delta_{h} v+\nu_{1 z} \partial_{z z}^{2} v, \\
\delta^{2} \frac{D w}{D t}-\frac{\lambda}{\varepsilon} u+\frac{\rho}{\varepsilon} & =-\frac{1}{\varepsilon} \partial_{z} \phi+\nu_{2 h} \Delta_{h} w+\nu_{2 z} \partial_{z z}^{2} w \\
\partial_{x} u+\partial_{y} v+\partial_{z} w & =0 \\
\frac{D \rho}{D t}+\frac{w}{\varepsilon} \partial_{z} \bar{\rho} & =\nu_{3 h} \Delta_{h} \rho+\nu_{3 z} \partial_{z z}^{2} \rho
\end{aligned}
$$

where we recall that $\varepsilon$ is the Rossby number (meant to go to zero), $\delta=H / L$ is the domain aspect ratio, and $\lambda=\delta \cot \theta_{0}$. The operator $\Delta_{h}$ is the horizontal Laplacian. In those equations, we do not consider any external forcing, except diffusion terms with kinematic viscosities $\left(\nu_{1} \bullet, \nu_{2} \bullet>0\right)$ and with eddy viscosities $\left(\nu_{3} \bullet>0\right)$ that are required for geophysical flows (see e.g. [7], Chap. 3, p. 39).

When we considered the scaling numbers introduced above, we have implicitly assumed that the leading term at the left-hand-side of equation (2.1a) was $f v$, which means (when comparing the two Coriolis terms in this equation) that $\delta \cot \theta_{0}$ should not be too large:

$$
\lambda \lesssim 1
$$

Fortunately, because the aspect ratio $\delta=H / L$ is rather small in large ocean models, the condition (2.3) is easily satisfied.

Remark 2.1. Actually, the term $\delta^{2} D w / D t$ could be set to zero in (2.2c) above with no modification in the sequel: indeed the reader will see below that $w$ vanishes at the main order, hence $\delta^{2} D w / D t=O(\varepsilon)$ can be neglected prior to the QG approximation.

The final model is thus called QHQG since the differences between the new QG model and the traditional one rely only on the terms related to the Coriolis force.

The key point of the present work is to draw the reader's attention on the fact that the ratio $\lambda / \varepsilon=\cot \theta_{0} \delta / \varepsilon$ is not necessarily small compared to $1 / \varepsilon$, and that it may have some physical impact (see Sect. 2.4).

\footnotetext{
${ }^{4}$ This assumption correspond to a small Froude number, of order $\varepsilon$.
} 


\subsubsection{Geostrophic balance}

We now consider an asymptotic expansion of all variables with respect to the Rossby number: for every unknown function $\gamma$, we write the formal asymptotic expansion

$$
\gamma=\gamma^{(0)}+\varepsilon \gamma^{(1)}+\varepsilon^{2} \gamma^{(2)}+\ldots
$$

where $\left(\gamma^{(j)}\right)_{j \geq 0}$ behave as $O(1)$ as $\varepsilon$ goes to zero. Equations (2.2a)-(2.2c) give, keeping only the leading order terms in $\varepsilon$ :

$$
\begin{aligned}
-v^{(0)}+\lambda w^{(0)} & =-\partial_{x} \phi^{(0)} \\
u^{(0)} & =-\partial_{y} \phi^{(0)} \\
-\lambda u^{(0)}+\rho^{(0)} & =-\partial_{z} \phi^{(0)} .
\end{aligned}
$$

We complement this system with equation (2.2e) (written to the order zero and since $\partial_{z} \bar{\rho}$ never vanishes) which leads to $w^{(0)}=0$.

Remark 2.2. An alternative way to obtain the main order of $w$ is based on the incompressibility contition. It reads $\partial_{z} w^{(0)}=-\partial_{x} u^{(0)}-\partial_{y} v^{(0)}$ and this traditionally leads to $w^{(0)}=0$, thanks to equations $(2.4 \mathrm{a}),(2.4 \mathrm{~b})$ and boundary conditions on $w$ (see [2]). Here, the incompressibility condition does not provide $\partial_{z} w^{(0)}=0$, but we have, denoting $\partial_{Z}=\partial_{z}+\lambda \partial_{y}$ :

$$
\begin{aligned}
\partial_{Z} w^{(0)} & =\partial_{z} w^{(0)}+\lambda \partial_{y} w^{(0)} \\
& =-\partial_{x} u^{(0)}-\partial_{y} v^{(0)}+\lambda \partial_{y} w^{(0)} \\
& =\operatorname{curl}\left(\partial_{y} \phi, \partial_{x} \phi\right) \\
& =0 .
\end{aligned}
$$

Thanks to $(2.5)$ and to homogeneous boundary conditions on $w^{(0)}$, we obtain again that $w^{(0)}=0$.

The geostrophic equations read:

$$
\begin{aligned}
-v^{(0)} & =-\partial_{x} \phi^{(0)}, \\
u^{(0)} & =-\partial_{y} \phi^{(0)}, \\
\rho^{(0)} & =-\partial_{z} \phi^{(0)}-\lambda \partial_{y} \phi^{(0)}=-\partial_{Z} \phi^{(0)}, \\
w^{(0)} & =0 .
\end{aligned}
$$

\subsubsection{Quasi-geostrophic equations}

Now we need the first order equations in order to determine the evolution of $\phi^{(0)}$. We denote by $d_{g}$ the zero-order material derivative:

$$
d_{g}=\partial_{t}+u^{(0)} \partial_{x}+v^{(0)} \partial_{y}
$$

The first order equations are:

$$
\begin{aligned}
d_{g} u^{(0)}-\beta_{0} y v^{(0)}-v^{(1)}+\lambda w^{(1)} & =-\partial_{x} \phi^{(1)}+\nu_{1 h} \Delta_{h} u^{(0)}+\nu_{1 z} \partial_{z z}^{2} u^{(0)}, \\
d_{g} v^{(0)}+\beta_{0} y u^{(0)}+u^{(1)} & =-\partial_{y} \phi^{(1)}+\nu_{1 h} \Delta_{h} v^{(0)}+\nu_{1 z} \partial_{z z}^{2} v^{(0)}, \\
-\lambda u^{(1)}+\rho^{(1)} & =-\partial_{z} \phi^{(1)} \\
\partial_{x} u^{(1)}+\partial_{y} v^{(1)}+\partial_{z} w^{(1)} & =0 \\
d_{g} \rho^{(0)}+w^{(1)} \partial_{z} \bar{\rho} & =\nu_{3 h} \Delta_{h} \rho^{(0)}+\nu_{3 z} \partial_{z z}^{2} \rho^{(0)} .
\end{aligned}
$$


We now take the curl of equations (2.7a) and (2.7b) to obtain, thanks to equation (2.7d)

$$
d_{g}\left(\partial_{x} v^{(0)}-\partial_{y} u^{(0)}\right)-\partial_{z} w^{(1)}-\lambda \partial_{y} w^{(1)}+\beta_{0} v^{(0)}=\nu_{1 h} \Delta_{h}\left(\partial_{x} v^{(0)}-\partial_{y} u^{(0)}\right)+\nu_{1 z} \partial_{z z}^{2}\left(\partial_{x} v^{(0)}-\partial_{y} u^{(0)}\right) .
$$

We notice, as for the traditional QG equations, that $\beta_{0} v^{(0)}=d_{g}\left(\beta_{0} y\right)$. We thus try to express the quantity $-\partial_{z} w^{(1)}-\lambda \partial_{y} w^{(1)}=-\partial_{Z} w^{(1)}$ as $d_{g}(\Gamma)$ plus viscous terms, where $\Gamma$ is a function to be defined. To this aim, we will extensively make use of equation $(2.7 \mathrm{e})$ that we reformulate:

$$
w^{(1)}=N^{-2}\left(d_{g} \rho^{(0)}-\nu_{3 h} \Delta_{h} \rho^{(0)}-\nu_{3 z} \partial_{z z}^{2} \rho^{(0)}\right)=d_{g}\left(N^{-2} \rho^{(0)}\right)-\nu_{3 h} N^{-2} \Delta_{h} \rho^{(0)}-\nu_{3 z} N^{-2} \partial_{z z}^{2} \rho^{(0)} .
$$

Given (2.9), we may compute the required quantity

$$
\begin{aligned}
\partial_{z} w^{(1)}+\lambda \partial_{y} w^{(1)}= & \partial_{z}\left(d_{g}\left(N^{-2} \rho^{(0)}\right)\right)+\lambda \partial_{y}\left(d_{g}\left(N^{-2} \rho^{(0)}\right)\right) \\
& -\nu_{3 h}\left(\partial_{z}+\lambda \partial_{y}\right)\left(N^{-2} \Delta_{h} \rho^{(0)}\right)-\nu_{3 z}\left(\partial_{z}+\lambda \partial_{y}\right)\left(N^{-2} \partial_{z z}^{2} \rho^{(0)}\right) .
\end{aligned}
$$

We remark that for any function $\gamma$ and any variable $*$ we have the identity

$$
\left(d_{g}(\gamma)\right)_{*}=d_{g}\left(\gamma_{*}\right)+u_{*}^{(0)} \partial_{x} \gamma+v_{*}^{(0)} \partial_{y} \gamma
$$

so that we can write equation $(2.10)$

$$
\begin{aligned}
\partial_{z} w^{(1)}+\lambda \partial_{y} w^{(1)}= & d_{g}\left(\partial_{z}\left(N^{-2} \rho^{(0)}\right)\right)+\lambda d_{g}\left(\partial_{y}\left(N^{-2} \rho^{(0)}\right)\right) \\
& -\nu_{3 h}\left(\partial_{z}+\lambda \partial_{y}\right)\left(N^{-2} \Delta_{h} \rho^{(0)}\right)-\nu_{3 z}\left(\partial_{z}+\lambda \partial_{y}\right)\left(N^{-2} \partial_{z z}^{2} \rho^{(0)}\right)+R,
\end{aligned}
$$

where the remainder $R$, according to the remark above, is

$$
\begin{aligned}
R & =\partial_{z} u^{(0)} \partial_{x}\left(N^{-2} \rho^{(0)}\right)+\partial_{z} v^{(0)} \partial_{y}\left(N^{-2} \rho^{(0)}\right)+\lambda \partial_{y} u^{(0)} \partial_{x}\left(N^{-2} \rho^{(0)}\right)+\lambda \partial_{y} v^{(0)} \partial_{y}\left(N^{-2} \rho^{(0)}\right) \\
& =\partial_{x}\left(N^{-2} \rho^{(0)}\right)\left(\partial_{z} u^{(0)}+\lambda \partial_{y} u^{(0)}\right)+\partial_{y}\left(N^{-2} \rho^{(0)}\right)\left(\partial_{z} v^{(0)}+\lambda \partial_{y} v^{(0)}\right) .
\end{aligned}
$$

Using equations (2.6a)-(2.6c) again, we have

$$
R=N^{-2} \partial_{x} \rho^{(0)} \partial_{y} \rho^{(0)}-N^{-2} \partial_{y} \rho^{(0)} \partial_{x} \rho^{(0)}=0,
$$

which simplifies equation (2.11) as follows:

$$
\begin{aligned}
\partial_{Z} w^{(1)}= & \partial_{z} w^{(1)}+\lambda \partial_{y} w^{(1)}=d_{g}\left(\partial_{z}\left(N^{-2} \rho^{(0)}\right)+\lambda \partial_{y}\left(N^{-2} \rho^{(0)}\right)\right) \\
& -\nu_{3 h}\left(\partial_{z}+\lambda \partial_{y}\right)\left(N^{-2} \Delta_{h} \rho^{(0)}\right)-\nu_{3 z}\left(\partial_{z}+\lambda \partial_{y}\right)\left(N^{-2} \partial_{z z}^{2} \rho^{(0)}\right) .
\end{aligned}
$$

Back to equation (2.8), we replace expression (2.12) and get:

$$
\begin{aligned}
d_{g}\left(\partial_{x} v^{(0)}-\right. & \left.\partial_{y} u^{(0)}-\partial_{z}\left(N^{-2} \rho^{(0)}\right)-\lambda \partial_{y}\left(N^{-2} \rho^{(0)}\right)+\beta_{0} y\right)=\nu_{1 h} \Delta_{h}\left(\partial_{x} v^{(0)}-\partial_{y} u^{(0)}\right) \\
& +\nu_{1 z} \partial_{z z}^{2}\left(\partial_{x} v^{(0)}-\partial_{y} u^{(0)}\right)-\nu_{3 h}\left(\partial_{z}+\lambda \partial_{y}\right)\left(N^{-2} \Delta_{h} \rho^{(0)}\right)-\nu_{3 z}\left(\partial_{z}+\lambda \partial_{y}\right)\left(N^{-2} \partial_{z z}^{2} \rho^{(0)}\right) .
\end{aligned}
$$

Let us now rewrite equation (2.13), expressing everything in terms of $\phi^{(0)}$. We have

$$
\begin{gathered}
\left(\partial_{t}-\partial_{y} \phi^{(0)} \partial_{x}+\partial_{x} \phi^{(0)} \partial_{y}\right) \zeta=\nu_{1 h} \Delta_{h} \Delta_{h} \phi^{(0)}+\nu_{1 z} \partial_{z z}^{2} \Delta_{h} \phi^{(0)} \\
+\nu_{3 h}\left(\partial_{z}+\lambda \partial_{y}\right)\left(N^{-2} \Delta_{h}\left(\partial_{z} \phi^{(0)}+\lambda \partial_{y} \phi^{(0)}\right)\right)+\nu_{3 z}\left(\partial_{z}+\lambda \partial_{y}\right)\left(N^{-2} \partial_{z z}^{2}\left(\partial_{z} \phi^{(0)}+\lambda \partial_{y} \phi^{(0)}\right)\right) \\
\quad \zeta=\Delta_{h} \phi^{(0)}+N^{-2}\left(\partial_{z}+\lambda \partial_{y}\right)^{2} \phi^{(0)}+\partial_{z} N^{-2}\left(\partial_{z}+\lambda \partial_{y}\right) \phi^{(0)}+\beta_{0} y .
\end{gathered}
$$


We could write this more compactly for $\Phi=\phi^{(0)}$ with $\partial_{Z}=\partial_{z}+\lambda \partial_{y}$ and get the following viscous quasihydrostatic quasi-geostrophic (QHQG) equation:

$$
\begin{aligned}
& \left(\partial_{t}-\partial_{y} \Phi \partial_{x}+\partial_{x} \Phi \partial_{y}\right)\left(\Delta_{h} \Phi+\partial_{Z}\left(N^{-2} \partial_{Z} \Phi\right)+\beta_{0} y\right)= \\
& \nu_{1 h} \Delta_{h} \Delta_{h} \Phi+\nu_{1 z} \partial_{z z}^{2} \Delta_{h} \Phi+\nu_{3 h} \partial_{Z}\left(N^{-2} \Delta_{h} \partial_{Z} \Phi\right)+\nu_{3 z} \partial_{Z}\left(N^{-2} \partial_{z z}^{2} \partial_{Z} \Phi\right) .
\end{aligned}
$$

One can thus easily recognize the traditional QG equation (see Eq. (2.23) in [2] without viscosity or equation (2.7) in [1]), except that the differential operator $\partial_{z}$ is replaced by the tilted one $\partial_{Z}=\partial_{z}+\lambda \partial_{y}$, see [14]. We recall here that $\lambda=\delta \cot \theta_{0}$ is proportional to the domain aspect ratio. In particular, we recover the traditional QG equation when setting $\lambda=0$ in equations (2.16). These equations are also called quasi-geostrophic equations for slanted rotation in [11].

Equation (2.16) complemented by adapted initial conditions and, eventually, boundary conditions, constitutes the QHQG model that we will study in the following.

\subsection{Existence of solutions to the inviscid QHQG}

In this section, we are interested in existence results for the inviscid quasi-hydrostatic quasi-geostrophic equation (Eq. (2.16) with vanishing viscosities $\nu_{1 h}, \nu_{1 z}$ and $\nu_{3 h}, \nu_{3 z}$ ):

$$
\left(\partial_{t}-\partial_{y} \Phi \partial_{x}+\partial_{x} \Phi \partial_{y}\right)\left(\Delta_{h} \Phi+\partial_{Z}\left(N^{-2} \partial_{Z} \Phi\right)+\beta_{0} y\right)=0 .
$$

We consider a periodic domain in the horizontal variables with rigid boundaries at the top and bottom. More precisely, we denote by $B=\Sigma \times(0, h)=\left(-\frac{1}{2}, \frac{1}{2}\right) \times\left(-\frac{1}{2}, \frac{1}{2}\right) \times(0, h)$ the spatial domain where we establish existence of solutions, with $t \in[0, T]$ (where $T>0$ ) the time interval.

We assume the solutions to be horizontally periodic with period 1 . The pressure potential $\Phi$ is defined by the QHQG equation (2.17) up to a constant at each time; then we add the following condition: $\int_{B} \Phi=0$. We define $\mathbf{u}=\left(u^{(0)}, v^{(0)}\right)$ the horizontal velocity at the leading order, we omit the (0) superscripts and rewrite the QHQG equation together with the geostrophic equations as:

$$
\begin{aligned}
v & =\partial_{x} \Phi, \\
u & =-\partial_{y} \Phi, \\
\rho & =-\partial_{z} \Phi-\lambda \partial_{y} \Phi, \\
\omega & :=\partial_{x} v-\partial_{y} u-\partial_{z}\left(N^{-2} \rho\right)-\lambda \partial_{y}\left(N^{-2} \rho\right) \quad \text { in } B, \\
\partial_{t} \omega+\mathbf{u} \cdot \nabla \omega & =-\beta_{0} v \quad \text { in } B \times[0, T],
\end{aligned}
$$

with the initial condition

$$
\omega(x, y, z, 0)=\omega_{0}(x, y, z)
$$

and the boundary conditions

$$
\rho=0 \quad \text { at } z=0 \text { and } z=h .
$$

Existence results are based on the following property:

Lemma 2.3. The partial differential operator $\mathcal{L}$ defined by

$$
\mathcal{L} \Phi:=\Delta_{h} \Phi+\partial_{z}\left(N^{-2}\left(\partial_{z} \Phi+\lambda \partial_{y} \Phi\right)\right)+\lambda \partial_{y}\left(N^{-2}\left(\partial_{z} \Phi+\lambda \partial_{y} \Phi\right)\right) \text { in } B
$$

is strictly elliptic. 
Proof. The function $N^{-2}(z)$ is assumed to be bounded away from 0 for $z \in[0, h]$.

We expand $\mathcal{L}$ as:

$$
\begin{aligned}
\mathcal{L} \Phi & =\partial_{x x}^{2} \Phi+\left(1+\lambda^{2} N^{-2}\right) \partial_{y y}^{2} \Phi+N^{-2} \partial_{z z}^{2} \Phi+2 \lambda N^{-2} \partial_{y z}^{2} \Phi+\lambda \partial_{z}\left(N^{-2}\right) \partial_{y} \Phi+\partial_{z}\left(N^{-2}\right) \partial_{z} \Phi \\
& =\sum_{i=1}^{3} \sum_{j=1}^{3} a_{i j} \partial_{i, j}^{2} \Phi+\sum_{i=1}^{3} b_{i} \partial_{i} \Phi,
\end{aligned}
$$

where:

$$
A=\left(a_{i j}\right)_{i, j=1, \ldots, 3}=\left(\begin{array}{ccc}
1 & 0 & 0 \\
0 & 1+\lambda^{2} N^{-2} & \lambda N^{-2} \\
0 & \lambda N^{-2} & N^{-2}
\end{array}\right) \text {, and } b=\left(b_{i}\right)_{i=1, \ldots, 3}=\left(0, \lambda \partial_{z}\left(N^{-2}\right), \partial_{z}\left(N^{-2}\right)\right)^{\top} .
$$

For all $\xi=\left(\xi_{1}, \xi_{2}, \xi_{3}\right)^{\top}$, we have, for almost every $z$ in $[0, h]$ :

$$
\begin{aligned}
\sum_{i=1}^{3} \sum_{j=1}^{3} a_{i j} \xi_{i} \xi_{j} & =\xi_{1}^{2}+\left(1+\lambda^{2} N^{-2}\right) \xi_{2}^{2}+N^{-2} \xi_{3}^{2}+2 \lambda N^{-2} \xi_{2} \xi_{3} \\
& \geq \xi_{1}^{2}+\frac{1}{2} \xi_{2}^{2}+\frac{1}{2 \lambda^{2}+N^{2}} \xi_{3}^{2} \quad \text { (thanks to Young inequality) } \\
& \geq \theta|\xi|^{2} \quad \text { with } \theta:=\min \left(\frac{1}{2}, \frac{1}{2 \lambda^{2}+N^{2}}\right)>0
\end{aligned}
$$

The condition $\theta>0$ is satisfied as soon as $\lambda>0$. In the case where $\lambda=0$ (traditional equation), one needs to assume that $N^{-2}$ is bounded away from zero (see [2]).

Lemma 2.3 generalizes the ellipticity result of [2] for any $\lambda \in \mathbb{R}$ and we can now obtain the same existence results. Indeed, system (2.18)-(2.24) only differs from the corresponding system in [2] in equations (2.20) and (2.21).

We first obtain the local existence of solutions:

Theorem 2.4 (Short-time existence of solutions to the QHQG model). If the initial vorticity $\omega_{0}$ is in $H^{s}(B)$ for some $s \geq 3$ with $\left|\omega_{0}\right|_{s} \leq M$, then there exists a time $T^{*}>0$ and a solution $\omega$ in $\mathcal{C}\left(\left[0, T^{*}\right], H^{s}(B)\right)$ to the QHQG model (2.18)-(2.24), where $T^{*}$ depends only on $M, B, \lambda$ and $\beta_{0}$. The vorticity $\omega$ satisfies the estimate $\|\omega\|_{s, T^{*}} \leq 2 M$.

Proof. The proof follows the lines of [2], considering an iterative process:

- starting from $\xi^{0}(x, y, z, t)=\omega_{0}(x, y, z)$ given,

- for $k \geq 0$ compute $\Phi^{k}$ thanks to the relation:

$$
\left\{\begin{array}{l}
\partial_{x x}^{2} \Phi^{k}+\partial_{y y}^{2} \Phi^{k}+\partial_{z}\left(\frac{\partial_{z} \Phi^{k}+\lambda \partial_{y} \Phi^{k}}{N^{2}}\right)+\frac{\lambda}{N^{2}} \partial_{y}\left(\partial_{z} \Phi^{k}+\lambda \partial_{y} \Phi^{k}\right)=\xi^{k} \text { in } B \\
\partial_{z} \Phi^{k}+\lambda \partial_{y} \Phi^{k}=0 \quad \text { for } z=0 \text { and } z=h \\
\int_{B} \Phi^{k}=0
\end{array}\right.
$$

- then set $u^{k}=-\partial_{y} \Phi^{k}$ and $v^{k}=\partial_{x} \Phi^{k}$,

- for every $z \in[0, h]$, compute $\xi^{k+1}$ solution of

$$
\partial_{t} \xi^{k+1}+\left(u^{k}, v^{k}\right)^{\top} \cdot \nabla \xi^{k+1}=-\beta_{0} v^{k},
$$

with $\xi^{k+1}(t=0)=\omega_{0}$, with periodic boundary conditions in $x$ and $y$. 
The elliptic result (Lem. 2.3) still gives the estimate:

$$
\left|\Phi^{k}\right|_{s+2} \leq C_{0}\left|\xi^{k}\right|_{s} \quad \text { with } C_{0}=C_{0}(B, N(z), \lambda)>0
$$

such that, equations (2.18), (2.19) and (2.22) being unchanged, the short-time existence of the $k$ th vorticity iterate $\xi^{k}$ follows, as well as the upper bound on its $H^{s}$ norm.

The end of the proof, for the convergence of the iterates $\left\{\xi^{k}(t)\right\}_{k \geq 0}$, is not modified by the $\lambda$ coefficient.

We are also able to adapt the proof of global solutions for the QG model by [2] to get:

Theorem 2.5 (Global existence of solutions to the QHQG model). If $\omega_{0}$ is in $H^{s}(B)$ for some $s \geq 3$, then given any time $T>0$, there exists a solution $\omega$ in $\mathcal{C}\left([0, T], H^{s}(B)\right)$ to the QHQG model (2.18)-(2.24).

Proof. From Lemma 2.3, relations (2.18)-(2.20) and from

$$
\left\{\begin{array}{l}
\partial_{x x}^{2} \Phi+\partial_{y y}^{2} \Phi+\partial_{z}\left(\frac{\partial_{z} \Phi+\lambda \partial_{y} \Phi}{N^{2}}\right)+\frac{\lambda}{N^{2}} \partial_{y}\left(\partial_{z} \Phi+\lambda \partial_{y} \Phi\right)=\omega \text { in } B \\
\partial_{z} \Phi+\lambda \partial_{y} \Phi=0 \quad \text { for } z=0 \text { and } z=h \\
\int_{B} \Phi=0
\end{array}\right.
$$

we have the elliptic estimate:

$$
|\mathbf{u}|_{s+1}+|\rho|_{s+1} \leq C|\omega|_{s}
$$

such that we also have a global estimate on the QHQG vorticity $\omega$ of the form

$$
|\omega(t)|_{s} \leq K(t) \quad \forall t \in[0, T],
$$

where the function $K$ only depends on the $H^{s}$ norm of the initial condition $\omega_{0}$. Thanks to the short-time existence, the global existence can be proved, using the global bound

$$
|\omega(t)|_{s} \leq \max _{t \in[0, T]} K(t) \quad \forall t \in[0, T]
$$

to reach any time $T$ thanks to an iterative process.

\subsection{Existence of solutions to the viscous QHQG}

As in Section 2.2, we are interested here in adapting existence results of [1] to the QHQG equation (2.16).

Let us consider the QHQG equation with vanishing vertical viscosities $\nu_{1 z}$ and $\nu_{3 z}$ :

$$
\left(\partial_{t}-\partial_{y} \Phi \partial_{x}+\partial_{x} \Phi \partial_{y}\right)\left(\Delta_{h} \Phi+\partial_{Z}\left(N^{-2} \partial_{Z} \Phi\right)+\beta_{0} y\right)=\nu_{1 h} \Delta_{h} \Delta_{h} \Phi+\nu_{3 h} \partial_{Z}\left(N^{-2} \Delta_{h} \partial_{Z} \Phi\right),
$$

in the spatial domain $B=(0,1) \times(0,1) \times(0, h)$, for $t \in[0, T]$ with the following boundary conditions at the top and at the bottom:

$$
\partial_{z} \Phi=0 \text { for } z=0 \text { and } z=h,
$$

and with periodic boundary conditions (with period 1 ) for the horizontal variables. The initial condition on the vorticity $\omega$ given by $\omega=\Delta_{h} \Phi+\partial_{Z}\left(N^{-2} \partial_{Z} \Phi\right)$ is:

$$
\omega(x, y, z, 0)=\omega_{I}(x, y, z),
$$

where $\omega_{I}$ is periodic with period 1 in the horizontal directions.

In the following we denote by $f \in \mathcal{C}^{n}(\bar{\Omega})$ a function that is 1-periodic in $x$ and $y$ and with all its derivatives (with respect to variables in $\Omega$ ) $\partial_{\bullet}^{\ell} f$ continuous on $\bar{\Omega}$ for $0 \leq \ell \leq n$. 
Theorem 2.6 (Existence of solution to the dissipative QHQG model). If $N(z)$ is continuous and continuously differentiable on $[0, h]$, if $\omega_{I}$ and its horizontal derivatives (up to the second order) are in $\mathcal{C}^{\gamma}(\bar{B})$, then there exists a unique solution $\left(\Phi^{*}, \omega^{*}\right)$ to the $Q H Q G$ problem $(2.26)-(2.28)$ in the time interval $\left[0, T^{*}\right]$, where $T^{*}$ is inversely proportional to the $\beta_{0}$ parameter, with:

- $\Phi^{*}$ has all its spatial partial derivatives $\partial_{\bullet}^{\ell} \Phi^{*}($ for $0 \leq \ell \leq 2)$ in $\mathcal{C}^{0}\left(\bar{B} \times\left[0, T^{*}\right]\right)$ and its second order spatial derivatives $\partial_{\bullet}^{2} \Phi^{*}$ in $\mathcal{C}^{\gamma}(\bar{B})$ uniformly in $t \in\left[0, T^{*}\right]$,

- $\omega^{*}$, its horizontal derivatives (up to the second order) and $\partial_{t} \omega^{*}$ are in $\mathcal{C}^{0}\left(\bar{B} \times\left[0, T^{*}\right]\right)$ and in $\mathcal{C}^{\gamma}(\bar{B})$ uniformly in $t \in\left[0, T^{*}\right]$.

Proof. The proof is exactly the one of [1], based on a Schauder fixed point for the existence and energy estimates for the uniqueness. As in Section 2.2, the only novelty for the QHQG model (2.26)-(2.28) is the $\partial_{Z}$ term in the vorticity instead of $\partial_{z}$ : Lemma 2.3 ensures the ellipticity of the corresponding operator and the rest of the proof remains unchanged. We refer to [1] for details and for the expression of $T^{*}$ in terms of $\beta$.

\subsection{Simple physical properties of the QHQG model}

We detail here some basic physical properties that can be obtained for the QHQG model and we perform comparisons with the well-known QG system.

We recall the QHQG equation (2.16):

$$
\begin{aligned}
&\left(\partial_{t}-\partial_{y} \Phi \partial_{x}+\partial_{x} \Phi \partial_{y}\right)\left(\Delta_{h} \Phi+\partial_{Z}\left(N^{-2} \partial_{Z} \Phi\right)+\beta_{0} y\right)= \\
& \nu_{1 h} \Delta_{h} \Delta_{h} \Phi+\nu_{1 z} \partial_{z z}^{2} \Delta_{h} \Phi+\nu_{3 h} \partial_{Z}\left(N^{-2} \Delta_{h} \partial_{Z} \Phi\right)+\nu_{3 z} \partial_{Z}\left(N^{-2} \partial_{z z}^{2} \partial_{Z} \Phi\right)
\end{aligned}
$$

where $\partial_{Z}=\partial_{z}+\lambda \partial_{y}$ and $\lambda=\delta \cot \theta_{0}$.

\subsubsection{Coordinate transformation}

We define a coordinate transform from $(\mathbf{x}, t)=(x, y, z, t)$ to $(\mathbf{X}, T)=(X, Y, Z, T)$ by

$$
X=x, \quad Y=y-\lambda z, \quad Z=z, \quad T=t .
$$

The transformed QHQG system is isomorphic to (2.16) because $\left(\partial_{t}-\partial_{y} \Phi \partial_{x}+\partial_{x} \Phi \partial_{y}\right)$ and $\Delta_{h}$, have identical functional forms in the transformed coordinates; $N^{2}(z)=N^{2}(Z)$; and $\beta y=\beta(Y+\lambda Z)$ can be replaced by $\beta Y$ because $\left(\partial_{t}-\partial_{y} \Phi \partial_{x}+\partial_{x} \Phi \partial_{y}\right)(\lambda Z)=0$. Thus, all QG solutions $\Phi(\mathbf{x}, t)$ are also QHQG solutions $\Phi(\mathbf{X}, T)$ if the initial and boundary conditions are consistent. Initial conditions are equivalently specified at $t=0$ or $T=0$. Planar vertical boundary surfaces at $z=z_{0}$ are also planar at $Z=z_{0}$. The same is true in $x$ and $X$. Only in $y$ is a planar surface in $y$ no longer planar in $Y$; therefore, the simple equivalence is for solutions that have an unbounded $y$ domain, or are $y$-periodic, or decay away in $y$ before the boundary.

\subsubsection{Separable solutions and vertical modes}

In the inviscid case (see Eq. (2.17)), QHQG has vertically separable solutions when linearized by neglecting $\left(\partial_{x} \Phi \partial_{y}-\partial_{y} \Phi \partial_{x}\right)\left(\Delta_{h} \Phi+\partial_{Z}\left(N^{-2} \partial_{Z} \Phi\right)\right)$, even for general $N(z), \Phi=\Pi(X, Y, T) F(Z)$. (QG has an analogous property.) Such QHQG solutions have a vertically upward phase tilt in the $(y, z)$ plane with a slope of $\mathrm{d} z / \mathrm{d} y=$ $\lambda^{-1}$ relative to the $z$ axis. This direction is aligned with the full rotation vector. Many phenomena influenced by the non-traditional approximation (e.g., convection and centrifugal instability as reviewed in [12]) are known

to exhibit this type of phase tilt. If we assume constant density at the vertical boundaries, $F$ is determined by the $1 \mathrm{D}$ eigenvalue problem,

$$
\partial_{Z}\left(N^{-2} \partial_{Z} F\right)+\mathcal{R}^{-2} F=0, \quad \partial_{Z} F=0 \text { at } Z=Z_{0}, Z_{1} .
$$

The eigenvalue $\mathcal{R}$ is the horizontal radius of deformation. With $N$ constant, the eigenmodes $F$ are cosine functions in $Z$. 


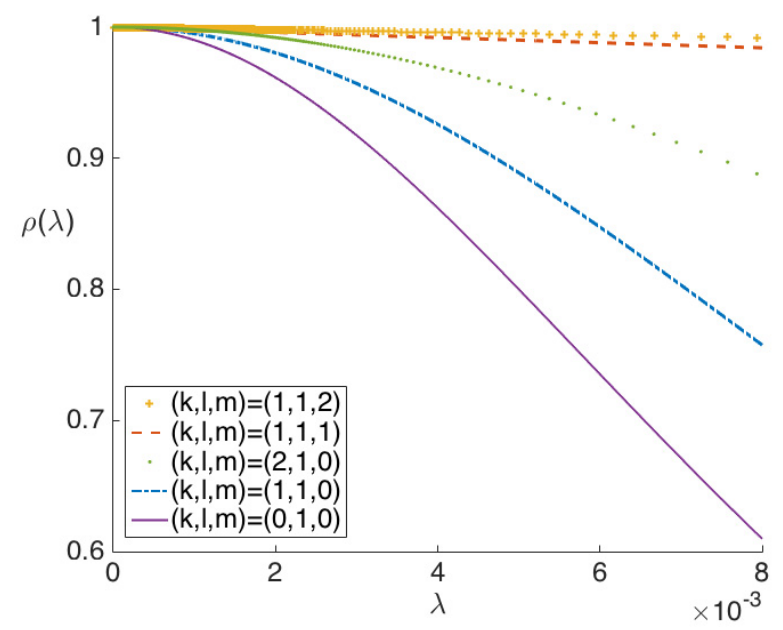

FiguRE 1. Values of $\rho_{k, l, m}(\lambda)$ for several values of $m$. The main differences can be seen with $m=0$. Other values of $k, l, m$ (in particular with $m>0$ ) do not bring any additional information. The computations are made with a typical value of the Brunt-Väisälä frequency $N=0.01$.

\subsubsection{Thermal wind balance}

The geostrophic equations (2.6a)-(2.6c) have a steady solution for a zonal flow. This can be expressed as $u=U(Y, Z), \varrho=-\int_{0}^{Z} N^{2}\left(Z^{\prime}\right) \mathrm{d} Z^{\prime}+B(Y, Z)$ with $\partial_{Z} U=-\partial_{Y} B$, which is thermal wind balance.

\subsubsection{Rossby wave modes}

One class of simple solutions is Rossby wave modes. They satisfy a linearized PDE usually justified by an assumption of small amplitude flow,

$$
\partial_{t}\left(\Delta_{h} \Phi+\left(\partial_{z}+\lambda \partial_{y}\right)\left(N^{-2}\left(\partial_{z}+\lambda \partial_{y}\right) \Phi\right)\right)+\beta \partial_{x} \Phi=0 .
$$

With constant $N$ in either a vertically bounded or unbounded domain, eigenmodes are

$$
\Phi \propto \mathrm{e}^{i(k x+l y+m z-\omega t)},
$$

with a dispersion relation,

$$
\omega=\frac{-\beta k}{K^{2}}, \quad K^{2}=k^{2}+l^{2}+\frac{1}{N^{2}}(m+\lambda l)^{2} .
$$

We can equivalently write this as

$$
\Phi \propto \mathrm{e}^{i(k X+l Y+M Z-\omega T)},
$$

with $M=m+\lambda l$.

Let us define the function

$$
\rho_{k, l, m}(\lambda)=\frac{\omega(\lambda)}{\omega(0)},
$$

that will evidence the difference between QG and QHQG models. We plot in Figure 1 above the behavior of $\rho_{k, l, m}(\lambda)$ as a function of $\lambda$ for various values of $k, l, m$.

Naturally, for $\theta_{0}=0$ we have a singularity $(\lambda=+\infty)$ and $\rho(\lambda)$ goes to zero. This advocates for the fact that QHQG and QG have drastically different behaviors "at the equator", where QG is known to be irrelevant. 
The results in Figure 1 show that the barotropic mode $m=0$ has a significantly lower frequency even for non-infinite (reasonable) values of $\lambda$. Indeed, assuming $l>0$ (otherwise $\rho=1$ ) a difference of $10 \%$ can be seen with $\lambda=4 \times 10^{-3}$ with $(k, l, m)=(0,1,0)$, which corresponds (with $\delta=10^{-3}$ which is a good typical value in the North Atlantic ocean) to $\theta_{0}=14^{\circ}$. This is not that close from the equator. For the mode $(k, l, m)=(1,1,0)$ the $10 \%$ margin leads to $\theta_{0}=11^{\circ}\left(\lambda=5 \times 10^{-3}\right)$.

Even if this $10 \%$ difference cannot be seen for $m>0$ (see Fig. 1), the barotropic mode is the more energetic one and this is another reason to claim that QH modeling should be used as much as possible.

\subsubsection{Vortex solutions}

A simple vortex solution is a nonlinear stationary state when $\beta=0$ and $N$ is constant. In QG this occurs for any axisymmetric profile, $\Phi(r, z)$, where $r=\sqrt{x^{2}+y^{2}}$ is the radial coordinate, which is sufficient to make $\left(\partial_{x} \Phi \partial_{y}-\partial_{y} \Phi \partial_{x}\right)\left(\Delta_{h} \Phi+\partial_{z}\left(N^{-2} \partial_{z} \Phi\right)\right)=0$ even when the arguments have large amplitude. Physical interest usually lies in profiles that are localized in $r, e . g$., a monopole with $\Phi(r)$ decaying away from a central extremum. In QHQG nonlinear stationary solutions exist for any profile $\Phi(R, Z)$, where $R^{2}=X^{2}+Y^{2}$. Thus, QH vortices are meridionally tilted rather than verically aligned. This tilted structure was previously proposed for Meddy vortices by [23].

\subsubsection{Fourier-space estimates for non-traditional effects}

Assuming a wavenumber-space characterization of the solution, as commonly done for turbulent flows, in terms of vertical and horizontal wavenumbers, $k v$ and $k h$, or equivalently local values of $H$ and $L$, we can ask when the $\lambda$ value is not small.

In QG the common view (sometimes called Charney's stretched isotropy) is that the Burger number, $B u=$ $N H / 2 \Omega L=k h /(2 \Omega k v / N)$, is order one while $2 \Omega / N$ is small. If $H / L \sim 2 \Omega / N \ll 1$, then $\lambda \sim(2 \Omega / N) \cot \theta_{0}$, which will be small except when $\theta_{0}$ is very close to the Equator.

Alternatively, if we consider flow patterns with $2 \Omega k v / N k h \sim r \ll 1$, then $\lambda \sim(2 \Omega / N r) \cot \theta_{0}$. For small enough $r, \lambda$ need not be small, and the QH correction to QG will be important. This happens when the aspect ratio $H / L$ is large, the stratification is weak, and/or $\theta_{0}$ is small. Large aspect ratio can be described from the QG perspective as atypically "tall" flows.

\section{Place of the nOntraditional MOdels}

The objective of this section is to show that the quasi-hydrostatic quasi-geostrophic equation (2.16) is an intermediate model between the non-hydrostatic equations and the usual (traditional) quasi-geostrophic equation, namely equation (2.16) with $\lambda=0$ (or equivalently $Z=z$ ).

\subsection{Hierarchy of models}

We can represent these models on the diagram of Figure 2.

The convergence of NH towards QHQG (see (1) in Fig. 2) is proved in [11]: based on techniques developed in [10], the authors prove that the solution of $\mathrm{NH}$ weakly converges, when $\varepsilon$ tends to zero, to a limit that satisfies QHQG. The convergence cannot be in the strong sense due to fast oscillations that can appear in NH and that disappear in QHQG.

The convergence of NHT (that are exactly NH with $\lambda=0$ ) to QG (that is (2) in Fig. 2) have been studied in several papers; let us mention [2] in a periodic domain in the horizontal variables, with regular initial data, or in [5] in a three dimensional periodic domain, for ill prepared data.

In the following of this section, we are interested in the right part (3) of the diagram 2: we prove that a solution of QHQG converges to a solution of QG when $\delta$ (and consequently $\lambda$ ) tends to zero. 


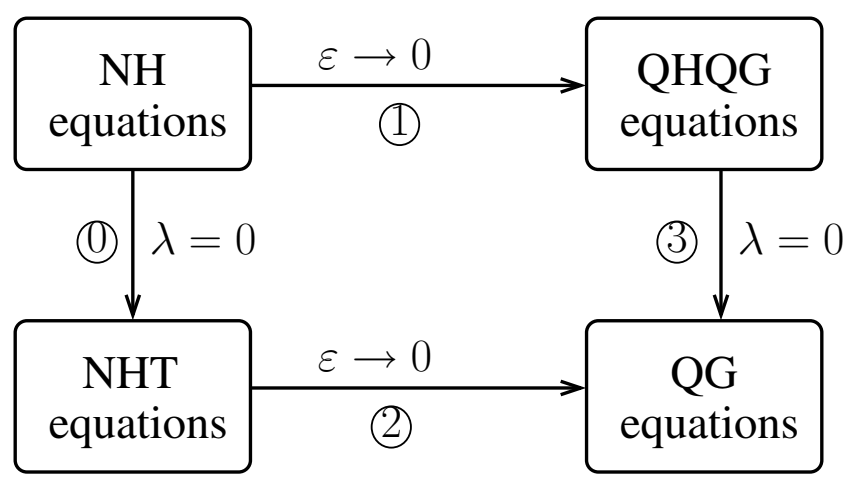

FiguRE 2. Non-hydrostatic equations and quasi-geostrophic equations: models with and without nontraditional terms. NH: non-hydrostatic equations, see (2.1); NHT: non-hydrostatic traditional equations, see (2.1) without the $f^{*}$ terms; QHQG: quasi-hydrostatic quasi-geostrophic equation, see (2.16); QG: (traditional) quasi-geostrophic equation, see (2.16) with $\lambda\left(=\delta \cot \theta_{0}\right)=0$.

\subsection{Convergence as $\delta$ (or $\lambda$ ) tends to zero}

We are interested in the limit when the aspect ratio $\delta$ tends to zero, which means that $\lambda$ also tends to zero. More precisely, we prove that the solution of the quasi-hydrostatic quasi-geostrophic model (2.16) converges to its corresponding hydrostatic model when the aspect ratio $\delta$ tends to 0 , that is, as $\lambda=\delta \cot \theta_{0}$, when the nontraditional Coriolis parameter $\lambda$ tends to 0 . We assume $\nu_{1 z}=0$ and $\nu_{3 z}$ of order $\varepsilon$ to be consistent with boundary conditions (see [17]).

Let us denote by $\phi^{\lambda}$ the solution of the nontraditional (quasi-hydrostatic) quasi-geostrophic model for a fixed $\lambda$ :

$$
\begin{aligned}
& \left(\partial_{t}-\partial_{y} \phi^{\lambda} \partial_{x}+\partial_{x} \phi^{\lambda} \partial_{y}\right)\left(\Delta_{h} \phi^{\lambda}+\partial_{Z}\left(N^{-2} \partial_{Z} \phi^{\lambda}\right)+\beta_{0} y\right)= \\
& \nu_{1 h} \Delta_{h} \Delta_{h} \phi^{\lambda}+\nu_{3 h} \partial_{Z}\left(N^{-2} \Delta_{h} \partial_{Z} \phi^{\lambda}\right)+\nu_{3 z} \partial_{Z}\left(N^{-2} \partial_{z z}^{2} \partial_{Z} \phi^{\lambda}\right) .
\end{aligned}
$$

in the domain $\Omega$ (where $\partial_{Z}=\partial_{z}+\lambda \partial_{y}$ ), with periodic conditions in $x$ and $y$, and verifying $\partial_{Z} \phi^{\lambda}=0$ at top $(z=h)$ and bottom $(z=0)$. At $t=0$, the function is given by $\phi_{0}^{\lambda}$. We assume the data to be well-prepared, that is $\phi_{0}^{\lambda} \rightarrow \phi_{0}$ when $\lambda \rightarrow 0$.

We want to prove that $\phi^{\lambda}$ converges when $\lambda \rightarrow 0$ towards a function $\phi$, solution of the following traditional (hydrostatic) quasi-geostrophic model:

$$
\begin{aligned}
\left(\partial_{t}-\partial_{y} \phi^{(G)} \partial_{x}+\partial_{x} \phi^{(G)} \partial_{y}\right)\left(\Delta_{h} \phi^{(G)}\right. & \left.+N^{-2} \partial_{z z}^{2} \phi^{(G)}+\partial_{z} N^{-2} \partial_{z} \phi^{(G)}+\beta_{0} y\right) \\
= & \nu_{1 h} \Delta_{h} \Delta_{h} \phi^{(G)}+\nu_{3 h} \partial_{z}\left(N^{-2} \Delta_{h} \partial_{z} \phi^{(G)}\right)+\nu_{3 z} \partial_{z}\left(N^{-2} \partial_{z z z}^{3} \phi^{(G)}\right)
\end{aligned}
$$

with $\partial_{z} \phi^{(G)}=0$ on the top and bottom, and $\phi^{(G)}(t=0)=\phi_{0}$.

\subsubsection{A priori estimates for $\phi^{\lambda}$}

Let us multiply equation (3.1) by $\phi^{\lambda}$ and integrate over the periodic domain (in $x$ and $y$ ) $\Omega$ :

$$
\int_{\Omega} \partial_{t}\left(\Delta_{h} \phi^{\lambda}+\partial_{Z}\left(N^{-2} \partial_{Z} \phi^{\lambda}\right)\right) \phi^{\lambda}=\nu_{1 h} \int_{\Omega} \Delta_{h} \Delta_{h} \phi^{\lambda} \phi^{\lambda}+\nu_{3 h} \int_{\Omega} \partial_{Z}\left(N^{-2} \Delta_{h} \partial_{Z} \phi^{\lambda}\right) \phi^{\lambda}+\nu_{3 z} \int_{\Omega} \partial_{Z}\left(N^{-2} \partial_{z z}^{2} \partial_{Z} \phi^{\lambda}\right) \phi^{\lambda}
$$


(due to the properties of the jacobian), which leads to:

$$
\begin{aligned}
-\frac{1}{2} \frac{\mathrm{d}}{\mathrm{d} t}\left\|\nabla_{h} \phi^{\lambda}\right\|_{2}^{2}-\frac{1}{2} \frac{\mathrm{d}}{\mathrm{d} t}\left\|N^{-1} \partial_{Z} \phi^{\lambda}\right\|_{2}^{2}= & \nu_{1 h}\left\|\Delta_{h} \phi^{\lambda}\right\|_{2}^{2}+\nu_{3 h}\left\|N^{-1} \nabla_{h} \partial_{Z} \phi^{\lambda}\right\|_{2}^{2}+\nu_{3 z}\left\|N^{-1} \partial_{z Z} \phi^{\lambda}\right\|_{2}^{2} \\
& +\int_{\Omega} \partial_{z} N^{-2} \partial_{z Z} \phi^{\lambda} \partial_{Z} \phi^{\lambda}
\end{aligned}
$$

that reads:

$$
\begin{array}{ll}
\nabla_{h} \phi^{\lambda} \text { is bounded in } L^{\infty}\left(0, T, L^{2}(\Omega)\right) & \text { if, } \forall \lambda>0,\left(\nabla_{h} \phi^{\lambda}\right)(t=0) \text { is in } L^{2}(\Omega), \\
\partial_{Z} \phi^{\lambda} \text { is bounded in } L^{\infty}\left(0, T, L^{2}(\Omega)\right) & \text { if, } \forall \lambda>0,\left(\partial_{Z} \phi^{\lambda}\right)(t=0) \text { is in } L^{2}(\Omega),
\end{array}
$$

and

$$
\begin{aligned}
& \Delta_{h} \phi^{\lambda} \text { is bounded in } L^{2}\left(0, T, L^{2}(\Omega)\right), \\
& \nabla \partial_{Z} \phi^{\lambda} \text { is bounded in } L^{2}\left(0, T, L^{2}(\Omega)\right),
\end{aligned}
$$

as the Brunt-Väisälä frequency $N(z)$ is bounded (from above and away from zero).

Let us also multiply equation (3.1) by $\Delta_{h} \phi^{\lambda}+\partial_{Z}\left(N^{-2} \partial_{Z} \phi^{\lambda}\right)$ and integrate over $\Omega$. We obtain:

$$
\begin{array}{r}
\int_{\Omega}\left(\partial_{t}\left(\Delta_{h} \phi^{\lambda}+\partial_{Z}\left(N^{-2} \partial_{Z} \phi^{\lambda}\right)\right)+\beta_{0} \partial_{x} \phi^{\lambda}\right)\left(\Delta_{h} \phi^{\lambda}+\partial_{Z}\left(N^{-2} \partial_{Z} \phi^{\lambda}\right)\right) \\
=\nu_{1 h} \int_{\Omega} \Delta_{h} \Delta_{h} \phi^{\lambda}\left(\Delta_{h} \phi^{\lambda}+\partial_{Z}\left(N^{-2} \partial_{Z} \phi^{\lambda}\right)\right)+\nu_{3 h} \int_{\Omega} \partial_{Z}\left(N^{-2} \Delta_{h} \partial_{Z} \phi^{\lambda}\right)\left(\Delta_{h} \phi^{\lambda}+\partial_{Z}\left(N^{-2} \partial_{Z} \phi^{\lambda}\right)\right) \\
+\nu_{3 z} \int_{\Omega} \partial_{z}\left(N^{-2} \partial_{z z}^{2} \partial_{Z} \phi^{\lambda}\right)\left(\Delta_{h} \phi^{\lambda}+\partial_{Z}\left(N^{-2} \partial_{Z} \phi^{\lambda}\right)\right)
\end{array}
$$

that gives:

$$
\begin{aligned}
& \frac{1}{2} \frac{\mathrm{d}}{\mathrm{d} t}\left\|\Delta_{h} \phi^{\lambda}+\partial_{Z}\left(N^{-2} \partial_{Z} \phi^{\lambda}\right)\right\|_{2}^{2}+\nu_{1 h}\left\|\nabla_{h} \Delta_{h} \phi^{\lambda}\right\|_{2}^{2}+\left(\nu_{1 h}+\nu_{3 h}\right)\left\|N^{-1} \Delta_{h} \partial_{Z} \phi^{\lambda}\right\|_{2}^{2} \\
&+\nu_{3 z}\left\|N^{-1} \nabla_{h} \partial_{z Z}^{2} \phi^{\lambda}\right\|_{2}^{2}+\nu_{3 h}\left\|\partial_{Z}\left(N^{-2} \nabla_{h} \partial_{Z} \phi^{\lambda}\right)\right\|_{2}^{2}+\nu_{3 z}\left\|\partial_{Z}\left(N^{-2} \partial_{z Z} \partial^{\lambda}\right)\right\|_{2}^{2} \\
&=-\beta_{0} \int_{\Omega} \partial_{x} \phi^{\lambda}\left(\Delta_{h} \phi^{\lambda}+\partial_{Z}\left(N^{-2} \partial_{Z} \phi^{\lambda}\right)\right)+\nu_{3 z} \int_{\Omega} \partial_{z} N^{-2} \partial_{z Z}^{2} \phi^{\lambda} \Delta_{h} \partial_{Z} \phi^{\lambda} .
\end{aligned}
$$

The r.h.s. can be bounded by $C_{1}+C_{2}\left\|\Delta_{h} \phi^{\lambda}+\partial_{Z}\left(N^{-2} \partial_{Z} \phi^{\lambda}\right)\right\|_{2}^{2}+C_{3}\left\|\Delta_{h} \partial_{Z} \phi^{\lambda}\right\|_{2}^{2}$ using the previous results, where the $C_{i}$ are constants, leading to:

$$
\begin{aligned}
\Delta_{h} \phi^{\lambda} \text { is bounded in } L^{\infty}\left(0, T, L^{2}(\Omega)\right) & \text { if, } \forall \lambda>0,\left(\Delta_{h} \phi^{\lambda}\right)(t=0) \text { is in } L^{2}(\Omega), \\
\partial_{Z}\left(N^{-2} \partial_{Z} \phi^{\lambda}\right) \text { is bounded in } L^{\infty}\left(0, T, L^{2}(\Omega)\right) & \text { if, } \forall \lambda>0,\left(\partial_{Z}\left(N^{-2} \partial_{Z} \phi^{\lambda}\right)\right)(t=0) \text { is in } L^{2}(\Omega), \\
N^{-1} \partial_{Z} \nabla_{h} \phi^{\lambda} \text { is bounded in } L^{\infty}\left(0, T, L^{2}(\Omega)\right) & \text { if, } \forall \lambda>0,\left(N^{-1} \partial_{Z} \nabla_{h} \phi^{\lambda}\right)(t=0) \text { is in } L^{2}(\Omega),
\end{aligned}
$$

and

$\nabla_{h} \Delta_{h} \phi^{\lambda}$ is bounded in $L^{2}\left(0, T, L^{2}(\Omega)\right)$,

$\partial_{Z} \Delta_{h} \phi^{\lambda}$ is bounded in $L^{2}\left(0, T, L^{2}(\Omega)\right)$,

$\partial_{Z}\left(N^{-2} \nabla \partial_{Z} \phi^{\lambda}\right)$ is bounded in $L^{2}\left(0, T, L^{2}(\Omega)\right)$,

$\partial_{z Z}^{2} \nabla_{h} \phi^{\lambda}$ is bounded in $L^{2}\left(0, T, L^{2}(\Omega)\right)$.

From equality (3.1), thanks to the previous properties, we also have:

$$
\partial_{t}\left(\Delta_{h} \phi^{\lambda}+\partial_{Z}\left(N^{-2} \partial_{Z} \phi^{\lambda}\right)\right) \text { is bounded in } L^{2}\left(0, T, H^{-1}(\Omega)\right) .
$$




\subsubsection{Convergence when $\lambda \rightarrow 0$.}

From the previous results, the gradient of $\phi^{\lambda}$ is in $L^{\infty}\left(0, T, L^{2}(\Omega)\right)$, so with Sobolev embeddings, $\phi^{\lambda}$ strongly converges to a limit denoted $\phi$ in $L^{\infty}\left(0, T, L^{p}(\Omega)\right)$, with $p<6$. Let us prove that $\phi$ satisfies the traditional quasi-geostrophic equation (3.2).

We just proved that $\partial_{t}\left(\Delta_{h} \phi^{\lambda}+\partial_{Z}\left(N^{-2} \partial_{Z} \phi^{\lambda}\right)\right)$ is bounded in $L^{2}\left(0, T, H^{-1}(\Omega)\right)$. With the previous results, we also have $\nabla\left(\Delta_{h} \phi^{\lambda}+\partial_{Z}\left(N^{-2} \partial_{Z} \phi^{\lambda}\right)\right)$ in $L^{2}\left(0, T, L^{2}(\Omega)\right)$ : thanks to Aubin-Lions theorem $\Delta_{h} \phi^{\lambda}+$ $\partial_{Z}\left(N^{-2} \partial_{Z} \phi^{\lambda}\right)$ strongly converges towards $\Delta_{h} \phi+\partial_{z}\left(N^{-2} \partial_{z} \phi\right)$ in $\mathcal{C}^{0}\left(0, T, L^{2}(\Omega)\right.$ ) (we recall that $\left.\partial_{Z}=\partial_{z}+\lambda \partial_{y}\right)$. So we can pass to the limit in the jacobian thanks to the strong convergence, the other terms do not bring any difficulty.

Theorem 3.1. Let $\phi^{\lambda}$ be a sequence of weak solutions of the nontraditional quasi-geostrophic equation (3.1) with initial data $\phi^{\lambda}(t=0)=\phi_{0}^{\lambda}$ satisfying $\phi_{0}^{\lambda} \rightarrow \phi_{0}$ in $L^{1}(\Omega)$ with, for all $\lambda>0$,

$$
\nabla \phi_{0}^{\lambda} \in L^{2}(\Omega), \quad \Delta \phi_{0}^{\lambda} \in L^{2}(\Omega), \text { and } \partial_{Z} \phi_{0}^{\lambda} \in L^{2}(\Omega) .
$$

Then, up to a subsequence, $\phi^{\lambda}$ strongly converges in $L^{\infty}\left(0, T, L^{p}(\Omega)\right)$, with $p<6$ towards $\phi$, weak solution of the quasi-geostrophic equation (3.2).

\section{Conclusion}

In this work we have shown that results of the reference paper [2] could be extended to quasi-hydrostatic modeling, leading to the QHQG equations (see also slanted or tilted models in [11,14]) with few adjustments. To the authors' opinion, this should advocate for the use of QHQG modeling rather than QG when the nontraditional parameter $\lambda$ is not small (in particular close to the equator). However, the convergence studies confirm that QG is a relevant approximation of QHQG for small $\lambda$ configurations. A numerical study to quantify more precisely the effect of the traditional approximation could be performed but it is beyond the scope of this work.

Acknowledgements. This work was supported by ANR through contract ANR-11-MONU-005 (COMODO). The authors are grateful to the anonymous referees for their suggestions and comments that widely improved the manuscript. The first author would also like to thank Philippe Grillot for helpful discussion related to this work.

\section{REFERENCES}

[1] A.F. Bennett and P.E. Kloeden, The dissipative quasigeostrophic equations. Mathematika 28 (1981) 265-285.

[2] A.J. Bourgeois and J.T. Beale, Validity of the quasigeostrophic model for large-scale flow in the atmosphere and ocean. SIAM J. Math. Anal. 25 (1994) 1023-1068.

[3] F.J. Bretherton and M.J. Karweit, Mid-ocean mesoscale modeling. In Numerical Models of Ocean Circulation. Ocean Affairs Board, National Research Council, National Academy of Sciences, Washington, DC (1975) 237-249.

[4] J.G. Charney, Geostrophic turbulence. J. Atmos. Sci. 28 (1971) 1087-1095.

[5] F. Charve, Convergence of weak solutions for the primitive system of the quasigeostrophic equations. Asymptot. Anal. 42 (2005) 173-209.

[6] A. Colin de Verdière and R. Schopp, Flows in a rotating spherical shell: the equatorial case. J. Fluid Mech. 276 (1994) $233-260$.

[7] B. Cushman-Roisin, Introduction to Geophysical Fluid Dynamics. Prentice Hall (1994).

[8] P.J. Dellar, Variations on a beta-plane: derivation of non-traditional beta-plane equations from Hamilton's principle on a sphere. J. Fluid Mech. 674 (2011) 174-195.

[9] C. Eckart, Hydrodynamics of oceans and atmospheres. Pergamon Press, New York (1960).

[10] P.F. Embid and A.J. Majda, Averaging over fast gravity waves for geophysical flows with arbitrary potential vorticity Commun. Partial Differ. Eq. 21 (1996) 619-658.

[11] P.F. Embid and A.J. Majda, Low Froude number limiting dynamics for stably stratified flow with small or finite Rossby numbers Geophys. Astrophys. Fluid Dyn. 87 (1998) 1-30.

[12] T. Gerkema, J.T.F. Zimmerman, L.R.M. Maas, H. van Haren, Geophysical and astrophysical fluid dynamics beyond the traditional approximation. Rev. Geophys. 46 (2008) 05.

[13] E. Grenier and N. Masmoudi, Ekman layers of rotating fluids, the case of well prepared initial data. Commun. Partial Differ. Eq. 22 (1997) 953-975. 
[14] K. Julien, E. Knobloch, R. Milliff and J. Werne, Generalized quasi-geostrophy for spatially anisotropic rotationally constrained flows. J. Fluid Mech. 555 (2006) 233-274.

[15] C. Lucas, M. Petcu and A. Rousseau, Quasi-hydrostatic primitive equations for ocean global circulation models. Chinese Ann. Math. B 31 (2010) 1-20.

[16] C. Lucas and A. Rousseau, New developments and cosine effect in the viscous Shallow-Water and quasi-geostrophic equations. SIAM Multiscale Model. Simul. 7 (2008) 796-813.

[17] J.C. McWilliams, A note on a consistent quasigeostrophic model in a multiply connected domain. Dynamics of Atmospheres and Oceans 1 (1977) 427-441.

[18] N. Masmoudi, Ekman layers of rotating fluids: The case of general initial data. Commun. Pure Appl. Math. 53 (2000) $432-483$.

[19] L. Perelman J. Marshall, C. Hill and A. Adcroft, Hydrostatic, quasi-hydrostatic, and nonhydrostatic ocean modeling. J. Geophys. Res. 102 (1997) 5733-5752.

[20] N.A. Phillips, The equations of motion for a shallow rotating atmosphere and the "traditional approximation". J. Atmospheric Sci. 23 (1966) 626-628.

[21] N.A. Phillips, Reply (to George Veronis). J. Atmospheric Sci. 25 (1968) 1155-1157.

[22] W.H. Raymond, Equatorial Meridional Flows: Rotationally Induced Circulations. Pure Appl. Geophys. 157 (2000) $1767-1779$.

[23] I.P. Semenova and L.N. Slezkin, Dynamically equilibrium shape of intrusive vortex formations in the ocean. Fluid Dynamics 38 (2003) 663-669.

[24] V.A. Sheremet, Laboratory experiments with tilted convective plumes on a centrifuge: a finite angle between the buoyancy force and the axis of rotation. J. Fluid Mech. 506 (2004) 217-244.

[25] F. Straneo, M. Kawase and S.C. Riser, Idealized models of slantwise convection in a baroclinic flow. J. Phys. Oceanogr. 32 (2002) 558-572.

[26] G. Veronis, Comments on Phillips' proposed simplification of the equations of motion for a shallow rotating atmosphere. $J$. Atmospheric Sci. 25 (1968) 1154-1155.

[27] G. Veronis, Large scale ocean circulation. Adv. Appl. Mech. 13 (1973) 1-92.

[28] R.K. Wangsness, Comments on the equations of motion for a shallow rotating atmosphere and the 'traditionnal approximation'. J. Atmospheric Sci. 27 (1970) 504-506.

[29] A.A. White and R.A. Bromley, Dynamically consistent quasi-hydrostatic equations for global models with a complete representation of the Coriolis force. Quarterly J. Roy. Meteorol. Soc. 121 (1995) 399-418.

[30] A.A. White, B.J. Hoskins, I. Roulstone and A. Staniforth, Consistent approximate models of the global atmosphere: shallow, deep, hydrostatic, quasi-hydrostatic and non-hydrostatic. Quarterly J. Roy. Meteorol. Soc. 131 (2005) $2081-2107$.

[31] A. Wirth and B. Barnier, Tilted convective plumes in numerical experiments. Ocean Model. 12 (2006) $101-111$. 